\title{
On a Class of Discrete Max-Type Difference Equation Model of Order Four
}

\author{
A. M. Alotaibi $\mathbb{D}^{1},{ }^{1}$ Abdul Khaliq $\mathbb{D}^{2},{ }^{2}$ Muhammad Ali, ${ }^{2}$ and Stephen Sadiq ${ }^{3}$ \\ ${ }^{1}$ Department of Mathematics, Faculty of Science, University of Tabuk, P.O. Box 741, Tabuk 71 491, Saudi Arabia \\ ${ }^{2}$ Department of Mathematics, Riphah Institute of Computing and Applied Sciences, Riphah International University \\ Lahore, Pakistan \\ ${ }^{3}$ Department of Mathematics, Minhaj University, Lahore, Pakistan
}

Correspondence should be addressed to Abdul Khaliq; khaliqsyed@gmail.com

Received 3 August 2021; Revised 23 December 2021; Accepted 6 January 2022; Published 29 January 2022

Academic Editor: A. E. Matouk

Copyright (c) 2022 A. M. Alotaibi et al. This is an open access article distributed under the Creative Commons Attribution License, which permits unrestricted use, distribution, and reproduction in any medium, provided the original work is properly cited.

\begin{abstract}
Discrete models are versatile and effective tools for assessing and addressing a wide range of scientific and practical problems. As a type of discrete model, the difference equation model has been widely employed in domains such as algorithm analysis, signal processing, biology, economics, and computer science. The global dynamical behavior of a class of discrete models known as the max-type difference equation model is the subject of the research. The purpose of this work is to look into the behavior of the solution of the following four-order max-type difference equation: $z_{i+1}=\max \left\{A_{i} / z_{i}, z_{i-3}\right\}, i=0,1,2, \ldots$
\end{abstract}

\section{Introduction}

This is generally known: life is intimately connected to mathematics, and science is intrinsically linked to mathematics. One of the core ideas in connecting practical research with mathematics is the formation of equations. Discrete models are useful and effective instruments for assessing and solving a wide range of scientific and practical issues (see [1-4]). A recurrence relation that comprises the unknown function and its difference but not the derivative is called a difference equation. It is a strong modeling tool for describing real-world discrete-time systems. The difference equation model, for example, is widely used in algorithm analysis, control theory, computer science, biology, economics, and physics, among other subjects. Many fluid mathematical models can be transformed into discrete forms to improve numerical simulations, which can make the study process and findings easier to understand. As a result, difference equation models can be used to represent a wide range of natural and social phenomena (see [4-12]). The majority of the time, the condition parameters vary or change within a particular range, necessitating the adjustment of the equation model. Parameter values are subject to imperfections in an equation model that explains biological phenomena, for example, due to specific modifications [13]. The condition value of the qualifying product is a range rather than a fixed value in some quality control situations. Mozaffari proposed an intelligent framework for determining the exhaust gas temperature (T ex) and hydrocarbon emissions (HC raw) from an automobile engine during cold start operation in [14]. The conditional parameter values are unknown since the cold start operation is treated as a temporary and uncertain phenomenon, thus the adaptive neuro-fuzzy inference computation and fuzzy logic controller are employed in the work. As a result, equation models with manipulated variables and maximum or minimum type equation models are more important; they are commonly used in electronic engineering, computer, artificial intelligence, and science. In [15], Li investigated the stability of particle trajectories in particle swarms using a difference equation and Z-transform and discussed the effects of p-Best, g-Best, and randomness on particle trajectories. In [16], Zhu produced one-dimensional and twodimensional time evolution equation models of nonlinear effect between pixels, based on his research on nonlinear impact off a digital image pixel grid. In [17], Chen proposed 
an excellent real-time autofocus technique using the discrete difference equation forecasting models. The proposed technique improves focusing speed. In [18], Fan used a difference equation model to examine a class of discrete SEIRS modeling techniques with general nonlinear occurrence and a discrete SEIRS epidemic model with standard occurrence. The condition that the sickness is permanent or that the model has a unique endemic equilibrium that is globally appealing is given by this equation model. There has been a lot of interest in investigating the dynamics of the max-type difference equation model in few years (see [19-25]). These findings are not only useful in and of themselves, but they can also provide insight into their disparate equivalents. The study of max-type difference equations has recently gained a lot of interest. Difference equations of this type can be found in several forms in automatic control systems. At the start of their analysis of these equations, scientists concentrated on the behavior of a few specific situations of the given difference equation:

$$
z_{i}=\max \left\{\frac{A_{i}^{(1)}}{z_{i-1}}, \frac{A_{i}^{(2)}}{z_{i-2}}, \ldots, \frac{A_{i}^{(j)}}{z_{i-i}}\right\}, \quad i \in \mathbb{N}_{0},
$$

where $i \in \mathbb{N}_{0}, A_{j}^{(n)}, n=1,2, \ldots, i$, are the real sequences, as well as the starting values $z_{-1}, z_{-2}, \ldots, z_{-i}$ are not equal to zero. Cinar and Yalcinkaya [26] focused on positive difference equation solutions.

$$
z_{i+1}=\max \left\{\frac{A}{z_{i}^{2}}, \frac{B z_{i-1}}{z_{i} z_{i-2}^{2}}\right\} .
$$

In a recent paper [27], it was demonstrated that every solution of the four-order max-type difference (10) with arbitrary nonzero real values as initial conditions and $A_{i}=$ constant $\in R$ is ultimately periodic with period four. In addition, we demonstrated in [28] every positive solution to the identical four-order nonautonomous max-type difference (10), where $A_{i}$ is a four-periodic sequence of real numbers with period four. The same result was obtained using a min-type difference equation for the similar equation. Simsek et al. [29] explored the answers to the difference equation as follows:

$$
z_{i+1}=\max \left\{z_{i-1}, \frac{1}{z_{i-1}}\right\} .
$$

Simsek et al. [30] also looked at how the solutions of the following system of difference equations behaved:

$$
\begin{aligned}
z_{i+1} & =\max \left\{\frac{A}{z_{i}}, \frac{t_{i}}{z_{i}}\right\}, \\
t_{i+1} & =\max \left\{\frac{A}{t_{i}}, \frac{z_{i}}{t_{i}}\right\} .
\end{aligned}
$$

Stevic [31] investigated the boundedness and global attractivity of positive difference equation solutions.

$$
z_{i+1}=\max \left\{c, \frac{z_{i}^{s}}{z_{i-1}^{s}}\right\} .
$$

In [32], the periodic nature of the solution of the maxtype difference equation was examined.

$$
z_{i+1}=\max \left\{\frac{z_{i}, A}{z_{i}^{2} z_{i-1}}\right\} .
$$

In [33], the authors propose, for the sake of dialogue, that the nonautonomous reciprocal max-type difference equation,

$$
x_{n+1}=\max \left\{\frac{A_{n}^{(0)}}{x_{n}}, \frac{A_{n}^{(1)}}{x_{n-1}}, \ldots, \frac{A_{n}^{(k)}}{x_{n-k}}\right\}, \quad n=0,1, \ldots,
$$

where the parameters $\left\{A_{n}^{(i)}\right\}_{n=0}^{\infty}$ are positive periodic sequences with periods $p_{i} \in 1,2, \ldots$ and the initial conditions are positive, when $k=1$ may serve as a phenomenological model of seizure activity as occurring in mesial (or middle) temporal lobe epilepsy. When $k=1$, the above equation may be written as

$$
x_{n+1}=\max \left\{\frac{A_{n}}{x_{n}}, \frac{B_{n}}{x_{n-1}}\right\}, \quad n=0,1, \ldots
$$

Authors ask the following questions: Why use a difference equation at all to model particular physiological processes of the human brain? Human brain function, from that which is physiological to that which is higher-level cognitive, can be viewed as recursive in nature. See, for example, the book by Corballis [34]. Why use the max-type difference equation, (9), to model normal functions of the human brain (e.g., the formation of memory) and abnormal function of the human brain (specifically, seizures)?

Motivated by above, the purpose of this work is to look into the behavior of the solution of the following four-order max-type difference equation:

$$
z_{i+1}=\max \left\{\frac{A_{i}}{z_{i}}, z_{i-3}\right\}, \quad i=0,1,2, \ldots
$$

The initial conditions $z_{-3}, z_{-2}, z_{-1}, z_{0}$ are arbitrary positive real values, and $\left\{A_{i}\right\}_{i=0}^{\infty}$ is a four-periodic sequence.

\section{Main Results}

First, we will add some basic definitions here and then will investigate two main theorems with their subcases.

Definition 1. A sequence $\left\{z_{i}\right\}_{i=-j}^{k}$ is said to be subsequently periodic, have period $p$, if there is $i_{0} \in\{-j, \ldots,-1,0,1, \ldots\}$ such that $z_{i}$ for all $i \geq i_{0}$. If $i_{0}=-i$, then we can say that the sequence $\left\{z_{j}\right\}_{j=-i}^{\infty}$ is periodic with period $s$.

Remark 2. Note that if $A_{i}=0$, equation (1) becomes $z_{i+1}=z_{i-3}$, implying that every solution has a period of four. As a result, we will look at the case $A_{i} \neq 0$ in the next section.

Remark 3. It should be noted that if $A_{i}=A$, equation (9) becomes $z_{i+1}=\max \left\{A / z_{i}, z_{i-4}\right\}$, and it has been demonstrated that this equation is recursive with period four. As a result, in the next section, we will look at the condition $A_{j}=\left\{A_{0}, A_{1}, A_{0}, A_{1}, A_{0}, A_{1}, \ldots\right\}, A_{1} \neq A_{0}$. 
Theorem 4. Consider the difference equation (9), where $A_{0}>A_{1}$. Then, at some point, every solution of equation (9) is periodic with period four.

Proof. From equation (9), we have

$$
\begin{aligned}
z_{i+1} & =\max \left\{\frac{A_{i}}{z_{i}}, z_{i-3}\right\}, i=0,1,2,3, \ldots, \\
z_{1} & =\max \left\{\frac{A_{0}}{z_{0}}, z_{-3}\right\} .
\end{aligned}
$$

We consider the following two cases: case $\left(A_{1}\right)$ : if $z_{-3} z_{0}<A_{0}$, then $z_{1}=A_{0} / z_{0}$ and

$$
z_{2}=\max \left\{\frac{A_{1}}{z_{1}}, z_{-2}\right\}=\max \left\{\frac{A_{1} z_{0}}{A_{0}}, z_{-2}\right\} \text {. }
$$

$\left(A_{11}\right):$ if $z_{-2} A_{0}>A_{1} z_{0}$, then $z_{2}=z_{-2}$ and

$$
z_{3}=\max \left\{\frac{A_{2}}{z_{2}}, z_{-1}\right\}=\max \left\{\frac{A_{0}}{z_{-2}}, z_{-1}\right\} .
$$

$\left(A_{111}\right):$ if $z_{-2} z_{-1}<A_{0}$, then $z_{3}=A_{0} / z_{-2}$ and

$$
z_{4}=\max \left\{\frac{A_{3}}{z_{3}}, z_{0}\right\}=\max \left\{\frac{A_{1} z_{-2}}{A_{0}}, z_{0}\right\} .
$$

$\left(A_{1111}\right):$ if $A_{0} z_{0}>A_{1} z_{-2}$, then $z_{4}=z_{0}$ and

$$
\begin{aligned}
& z_{5}=\max \left\{\frac{A_{4}}{z_{4}}, z_{1}\right\}=\max \left\{\frac{A_{0}}{z_{0}}, \frac{A_{0}}{z_{0}}\right\}=\frac{A_{0}}{z_{0}}, \\
& z_{6}=\max \left\{\frac{A_{5}}{z_{5}}, z_{2}\right\}=\max \left\{\frac{A_{1} z_{0}}{A_{0}}, z_{-2}\right\}=z_{-2} \\
& z_{7}=\max \left\{\frac{A_{6}}{z_{6}}, z_{3}\right\}=\max \left\{\frac{A_{0}}{z_{-2}}, \frac{A_{0}}{z_{-2}}\right\}=\frac{A_{0}}{z_{-2}} \\
& z_{8}=\max \left\{\frac{A_{7}}{z_{7}}, z_{4}\right\}=\max \left\{\frac{A_{1} z_{-2}}{A_{0}}, z_{0}\right\}=z_{0} \\
& z_{9}=\max \left\{\frac{A_{8}}{z_{8}}, z_{5}\right\}=\max \left\{\frac{A_{0}}{z_{0}}, \frac{A_{0}}{z_{0}}\right\}=\frac{A_{0}}{z_{0}}
\end{aligned}
$$

which is subsequently periodic with period four.

$$
\left\{z_{0}, \frac{A_{0}}{z_{0}}, z_{-2}, \frac{A_{0}}{z_{-2}}, z_{0}, \frac{A_{0}}{z_{0}} \ldots\right\} \text {. }
$$

$\left(A_{1112}\right):$ if $A_{0} z_{0}<A_{1} z_{-2}$, then $z_{4}=A_{1} z_{-2} / A_{0}$ and

$$
\begin{aligned}
& z_{5}=\max \left\{\frac{A_{4}}{z_{4}}, z_{1}\right\}=\max \left\{\frac{A_{0}^{2}}{A_{1} z_{-2}}, \frac{A_{0}}{z_{0}}\right\}=\frac{A_{0}^{2}}{A_{1} z_{-2}}, \\
& z_{6}=\max \left\{\frac{A_{5}}{z_{5}}, z_{2}\right\}=\max \left\{\frac{A_{1}^{2} z_{-2}}{A_{0}^{2}}, z_{-2}\right\}=z_{-2}, \\
& z_{7}=\max \left\{\frac{A_{6}}{z_{6}}, z_{3}\right\}=\max \left\{\frac{A_{0}}{z_{-2}}, \frac{A_{0}}{z_{-2}}\right\}=\frac{A_{0}}{z_{-2}}, \\
& z_{8}=\max \left\{\frac{A_{7}}{z_{7}}, z_{4}\right\}=\max \left\{\frac{A_{1} z_{-2}}{A_{0}}, z_{0}\right\}=z_{0}, \\
& z_{9}=\max \left\{\frac{A_{8}}{z_{8}}, z_{5}\right\}=\max \left\{\frac{A_{0}^{2}}{A_{1} z_{-2}}, \frac{A_{0}^{2}}{A_{1} z_{-2}}\right\}=\frac{A_{0}^{2}}{A_{1} z_{-2}},
\end{aligned}
$$

which is subsequently periodic with period four.

$$
\left\{\frac{A_{1} z_{-2}}{A_{0}}, \frac{A_{0}^{2}}{A_{1} z_{-2}}, z_{-2}, \frac{A_{0}}{z_{-2}}, z_{0}, \frac{A_{0}^{2}}{A_{1} z_{-2}}, \ldots\right\} .
$$

$\left(A_{112}\right):$ if $z_{-2} z_{-1}>A_{0}$, then $z_{3}=z_{-1}$ and

$$
\begin{aligned}
& z_{4}=\max \left\{\frac{A_{3}}{z_{3}}, z_{0}\right\}=\max \left\{\frac{A_{1}}{z_{-1}}, z_{0}\right\}=z_{0}, \\
& z_{5}=\max \left\{\frac{A_{4}}{z_{4}}, z_{1}\right\}=\max \left\{\frac{A_{0}}{z_{0}}, \frac{A_{0}}{z_{0}}\right\}=\frac{A_{0}}{z_{0}}, \\
& z_{6}=\max \left\{\frac{A_{5}}{z_{5}}, z_{2}\right\}=\max \left\{\frac{A_{1} z_{0}}{A_{0}}, z_{-2}\right\}=z_{-2}, \\
& z_{7}=\max \left\{\frac{A_{6}}{z_{6}}, z_{3}\right\}=\max \left\{\frac{A_{0}}{z_{-2}}, z_{-1}\right\}=z_{-1}, \\
& z_{8}=\max \left\{\frac{A_{7}}{z_{7}}, z_{4}\right\}=\max \left\{\frac{A_{1}}{z_{-1}}, z_{0}\right\}=z_{0}, \\
& z_{9}=\max \left\{\frac{A_{8}}{z_{8}}, z_{5}\right\}=\max \left\{\frac{A_{0}}{z_{0}}, \frac{A_{0}}{z_{0}}\right\}=\frac{A_{0}}{z_{0}},
\end{aligned}
$$

which is subsequently periodic with period four.

$$
\left\{z_{-1}, z_{0}, \frac{A_{0}}{z_{-2}}, z_{-2}, z_{-1}, z_{0}, \frac{A_{0}}{z_{0}}, \ldots\right\} \text {. }
$$

$\left(A_{12}\right):$ if $A_{0} z_{-2}<A_{1} z_{0}$, then $z_{2}=A_{1} z_{0} / A_{0}$ and

$$
z_{3}=\max \left\{\frac{A_{2}}{z_{2}}, z_{-1}\right\}=\max \left\{\frac{A_{0}^{2}}{A_{1} z_{0}}, z_{-1}\right\} .
$$

$\left(A_{121}\right):$ if $A_{0}^{2}<A_{1} z_{0} z_{-1}$, then $z_{3}=A_{0}^{2} / A_{1} z_{0}$ and 


$$
z_{4}=\max \left\{\frac{A_{3}}{z_{3}}, z_{0}\right\}=\max \left\{\frac{A_{1}^{2} z_{0}}{A_{0}^{2}}, z_{0}\right\} .
$$

$\left(A_{1211}\right)$ : if $A_{0}^{2} z_{0}>A_{1}^{2} z_{0}$, then $z_{4}=z_{0}$ and

$$
\begin{aligned}
& z_{5}=\max \left\{\frac{A_{4}}{z_{4}}, z_{1}\right\}=\max \left\{\frac{A_{0}}{z_{0}}, \frac{A_{0}}{z_{0}}\right\}=\frac{A_{0}}{z_{0}}, \\
& z_{6}=\max \left\{\frac{A_{5}}{z_{5}}, z_{2}\right\}=\max \left\{\frac{A_{1} z_{0}}{A_{0}}, \frac{A_{1} z_{0}}{A_{0}}\right\}=\frac{A_{1} z_{0}}{A_{0}}, \\
& z_{7}=\max \left\{\frac{A_{6}}{z_{6}}, z_{3}\right\}=\max \left\{\frac{A_{0}^{2}}{A_{1} z_{0}}, \frac{A_{0}^{2}}{A_{1} z_{0}}\right\}=\frac{A_{0}^{2}}{A_{1} z_{0}}, \\
& z_{8}=\max \left\{\frac{A_{7}}{z_{7}}, z_{4}\right\}=\max \left\{\frac{A_{1}^{2} z_{0}}{A_{0}^{2}}, z_{0}\right\}=z_{0}, \\
& z_{9}=\max \left\{\frac{A_{8}}{z_{8}}, z_{5}\right\}=\max \left\{\frac{A_{0}}{z_{0}}, \frac{A_{0}}{z_{0}}\right\}=\frac{A_{0}}{z_{0}},
\end{aligned}
$$

which is subsequently periodic with period four.

$$
\left\{z_{0}, \frac{A_{0}}{z_{0}}, \frac{A_{1} z_{0}}{A_{0}}, \frac{A_{0}^{2}}{A_{1} z_{0}}, z_{0}, \frac{A_{0}}{z_{0}}, \ldots\right\} .
$$

$\left(A_{1212}\right)$ : if $A_{0}^{2} z_{0}<A_{1}^{2} z_{0}$, then $z_{4}=A_{1}^{2} z_{0} / A_{0}^{2}$ and $z_{5}=\max \left\{\frac{A_{4}}{z_{4}}, z_{1}\right\}=\max \left\{\frac{A_{0}^{3}}{A_{1}^{2} z_{0}}, \frac{A_{0}}{z_{0}}\right\}=\frac{A_{0}}{z_{0}}$, $z_{6}=\max \left\{\frac{A_{5}}{z_{5}}, z_{2}\right\}=\max \left\{\frac{A_{1} z_{0}}{A_{0}}, \frac{A_{1} z_{0}}{A_{0}}\right\}=\frac{A_{1} z_{0}}{A_{0}}$, $z_{7}=\max \left\{\frac{A_{6}}{z_{6}}, z_{3}\right\}=\max \left\{\frac{A_{0}^{2}}{A_{1} z_{0}}, \frac{A_{0}^{2}}{A_{1} z_{0}}\right\}=\frac{A_{0}^{2}}{A_{1} z_{0}}$, $z_{8}=\max \left\{\frac{A_{7}}{z_{7}}, z_{4}\right\}=\max \left\{\frac{A_{1}^{2} z_{0}}{A_{0}^{2}}, \frac{A_{1}^{2} z_{0}}{A_{0}^{2}}\right\}=\frac{A_{1}^{2} z_{0}}{A_{0}^{2}}$, $z_{9}=\max \left\{\frac{A_{8}}{z_{8}}, z_{5}\right\}=\max \left\{\frac{A_{0}}{z_{0}}, \frac{A_{0}}{z_{0}}\right\}=\frac{A_{0}}{z_{0}}$,

which is subsequently periodic with period four.

$$
\left\{\frac{A_{1}^{2} z_{0}}{A_{0}^{2}}, \frac{A_{0}}{z_{0}}, \frac{A_{1} z_{0}}{A_{0}}, \frac{A_{0}^{2}}{A_{1} z_{0}}, \frac{A_{1}^{2} z_{0}}{A_{0}^{2}}, \frac{A_{0}}{z_{0}}, \ldots\right\} .
$$

$\left(A_{122}\right):$ if $A_{0}^{2}<A_{1} z_{0} z_{-1}$, then $z_{3}=z_{-1}$ and

$$
z_{4}=\max \left\{\frac{A_{3}}{z_{3}}, z_{0}\right\}=\max \left\{\frac{A_{1}}{z_{-1}}, z_{0}\right\} \text {. }
$$

$\left(A_{1221}\right):$ if $A_{1}>z_{-1} z_{0}$, then $z_{4}=A_{1} / z_{-1}$ and

$$
\begin{aligned}
& z_{5}=\max \left\{\frac{A_{4}}{z_{4}}, z_{1}\right\}=\max \left\{\frac{A_{0} z_{-1}}{A_{1}}, \frac{A_{0}}{z_{0}}\right\}=\frac{A_{0}}{z_{0}}, \\
& z_{6}=\max \left\{\frac{A_{5}}{z_{5}}, z_{2}\right\}=\max \left\{\frac{A_{1} z_{0}}{A_{0}}, \frac{A_{1} z_{0}}{A_{0}}\right\}=\frac{A_{1} z_{0}}{A_{0}}, \\
& z_{7}=\max \left\{\frac{A_{6}}{z_{6}}, z_{3}\right\}=\max \left\{\frac{A_{0}^{2}}{A_{1} z_{0}}, \frac{A_{0}^{2}}{A_{1} z_{0}}\right\}=\frac{A_{0}^{2}}{A_{1} z_{0}}, \\
& z_{8}=\max \left\{\frac{A_{7}}{z_{7}}, z_{4}\right\}=\max \left\{\frac{A_{1}^{2} z_{0}}{A_{0}^{2}}, \frac{A_{1}}{z_{-1}}\right\}=\frac{A_{1}}{z_{-1}}, \\
& z_{9}=\max \left\{\frac{A_{8}}{z_{8}}, z_{5}\right\}=\max \left\{\frac{A_{0}}{z_{0}}, \frac{A_{0}}{z_{0}}\right\}=\frac{A_{0}}{z_{0}},
\end{aligned}
$$

which is subsequently periodic with period four.

$$
\left\{\frac{A_{1}}{z_{-1}}, \frac{A_{0}}{z_{0}}, \frac{A_{1} z_{0}}{A_{0}}, \frac{A_{0}^{2}}{A_{1} z_{0}}, \frac{A_{1}}{z_{-1}}, \frac{A_{0}}{z_{0}}, \ldots\right\} .
$$

$\left(A_{1222}\right)$ : if $A_{1}<z_{-1} z_{0}$, then $z_{4}=z_{0}$ and

$$
\begin{aligned}
& z_{5}=\max \left\{\frac{A_{4}}{z_{4}}, z_{1}\right\}=\max \left\{\frac{A_{0}}{z_{0}}, \frac{A_{0}}{z_{0}}\right\}=\frac{A_{0}}{z_{0}}, \\
& z_{6}=\max \left\{\frac{A_{5}}{z_{5}}, z_{2}\right\}=\max \left\{\frac{A_{1} z_{0}}{A_{0}}, \frac{A_{1} z_{0}}{A_{0}}\right\}=\frac{A_{1} z_{0}}{A_{0}}, \\
& z_{7}=\max \left\{\frac{A_{6}}{z_{6}}, z_{3}\right\}=\max \left\{\frac{A_{0}^{2}}{A_{1} z_{0}}, z_{-1}\right\}=z_{-1}, \\
& z_{8}=\max \left\{\frac{A_{7}}{z_{7}}, z_{4}\right\}=\max \left\{\frac{A_{1}}{z_{-1}}, z_{0}\right\}=z_{0}, \\
& z_{9}=\max \left\{\frac{A_{8}}{z_{8}}, z_{5}\right\}=\max \left\{\frac{A_{0}}{z_{0}}, \frac{A_{0}}{z_{0}}\right\}=\frac{A_{0}}{z_{0}},
\end{aligned}
$$

which is subsequently periodic with period four.

$$
\left\{z_{0}, \frac{A_{0}}{z_{0}}, \frac{A_{1} z_{0}}{A_{0}}, z_{-1}, z_{0}, \frac{A_{0}}{z_{0}}, \ldots\right\} .
$$

Case $\left(A_{2}\right)$ : if $A_{0}<z_{-3} z_{0}$, then $z_{1}=z_{-3}$ and

$$
z_{2}=\max \left\{\frac{A_{1}}{z_{1}}, z_{-2}\right\}=\max \left\{\frac{A_{1}}{z_{-3}}, z_{-2}\right\} .
$$

$\left(A_{21}\right):$ if $A_{1}>z_{-3} z_{-2}$, then $z_{2}=A_{1} / z_{-3}$ and

$$
z_{3}=\max \left\{\frac{A_{2}}{z_{2}}, z_{-1}\right\}=\max \left\{\frac{A_{0} z_{-3}}{A_{1}}, z_{-1}\right\} .
$$

$\left(A_{211}\right):$ if $A_{0} z_{-3}>A_{1} z_{-1}$, then $z_{3}=A_{0} z_{-3} / A_{1}$ and 


$$
z_{4}=\max \left\{\frac{A_{3}}{z_{3}}, z_{0}\right\}=\max \left\{\frac{A_{1}^{2}}{A_{0} z_{-3}}, z_{0}\right\}=z_{0} .
$$

Since, $A_{0} z_{-3}>A_{1} z_{-1}$,

$$
\begin{aligned}
& z_{5}=\max \left\{\frac{A_{4}}{z_{4}}, z_{1}\right\}=\max \left\{\frac{A_{0}}{z_{0}}, z_{-3}\right\}=z_{-3}, \\
& z_{6}=\max \left\{\frac{A_{5}}{z_{5}}, z_{2}\right\}=\max \left\{\frac{A_{1}}{z_{-3}}, \frac{A_{1}}{z_{-3}}\right\}=\frac{A_{1}}{z_{-3}}, \\
& z_{7}=\max \left\{\frac{A_{6}}{z_{6}}, z_{3}\right\}=\max \left\{\frac{A_{0} z_{-3}}{A_{1}}, \frac{A_{0} z_{-3}}{A_{1}}\right\}=\frac{A_{0} z_{-3}}{A_{1}}, \\
& z_{8}=\max \left\{\frac{A_{7}}{z_{7}}, z_{4}\right\}=\max \left\{\frac{A_{1}^{2}}{A_{0} z_{-3}}, z_{0}\right\}=z_{0}, \\
& z_{9}=\max \left\{\frac{A_{8}}{z_{8}}, z_{5}\right\}=\max \left\{\frac{A_{0}}{z_{0}}, z_{-3}\right\}=z_{-3},
\end{aligned}
$$

which is subsequently periodic with period four.

$$
\left\{\frac{A_{0} z_{-3}}{A_{1}}, z_{0}, z_{-3}, \frac{A_{1}}{z_{-3}}, \frac{A_{0} z_{-3}}{A_{1}}, z_{0}, z_{-3}, \ldots\right\} \text {. }
$$

$\left(A_{212}\right):$ if $A_{0} z_{-3}<A_{1} z_{-1}$, then $z_{3}=z_{-1}$ and

$$
z_{4}=\max \left\{\frac{A_{3}}{z_{3}}, z_{0}\right\}=\max \left\{\frac{A_{1}}{z_{-1}}, z_{0}\right\}=z_{0} .
$$

Since, $z_{0} z_{-1}>A_{0}>A_{1}$,

$$
\begin{aligned}
& z_{5}=\max \left\{\frac{A_{4}}{z_{4}}, z_{1}\right\}=\max \left\{\frac{A_{0}}{z_{0}}, z_{-3}\right\}=z_{-3}, \\
& z_{6}=\max \left\{\frac{A_{5}}{z_{5}}, z_{2}\right\}=\max \left\{\frac{A_{1}}{z_{-3}}, \frac{A_{1}}{z_{-3}}\right\}=\frac{A_{1}}{z_{-3}}, \\
& z_{7}=\max \left\{\frac{A_{6}}{z_{6}}, z_{3}\right\}=\max \left\{\frac{A_{0} z_{-3}}{A_{1}}, z_{-1}\right\}=z_{-1}, \\
& z_{8}=\max \left\{\frac{A_{7}}{z_{7}}, z_{4}\right\}=\max \left\{\frac{A_{1}}{z_{-1}}, z_{0}\right\}=z_{0}, \\
& z_{9}=\max \left\{\frac{A_{8}}{z_{8}}, z_{5}\right\}=\max \left\{\frac{A_{0}}{z_{0}}, \frac{A_{0}}{z_{0}}\right\}=\frac{A_{0}}{z_{0}},
\end{aligned}
$$

which is subsequently periodic with period four.

$$
\left\{z_{-1}, z_{0}, z_{-3}, \frac{A_{1}}{z_{-3}}, z_{-1}, z_{0}, z_{-3}, \ldots\right\} \text {. }
$$

$\left(A_{22}\right)$ : if $A_{1}<z_{-3} z_{-2}$, then $z_{2}=z_{-2}$ and

$$
z_{3}=\max \left\{\frac{A_{2}}{z_{2}}, z_{-1}\right\}=\max \left\{\frac{A_{0}}{z_{-2}}, z_{-1}\right\} .
$$

$\left(A_{221}\right):$ if $A_{0}>z_{-2} z_{-1}$, then $z_{3}=A_{0} / z_{-2}$ and

$$
z_{4}=\max \left\{\frac{A_{3}}{z_{3}}, z_{0}\right\}=\max \left\{\frac{A_{1} z_{-2}}{A_{0}}, z_{0}\right\} .
$$

$\left(A_{2211}\right):$ if $A_{0} z_{0}>A_{1} z_{-2}$, then $z_{4}=z_{0}$ and

$$
\begin{aligned}
& z_{5}=\max \left\{\frac{A_{4}}{z_{4}}, z_{1}\right\}=\max \left\{\frac{A_{0}}{z_{0}}, z_{-3}\right\}=z_{-3}, \\
& z_{6}=\max \left\{\frac{A_{5}}{z_{5}}, z_{2}\right\}=\max \left\{\frac{A_{1}}{z_{-3}}, z_{-2}\right\}=z_{-2}, \\
& z_{7}=\max \left\{\frac{A_{6}}{z_{6}}, z_{3}\right\}=\max \left\{\frac{A_{0}}{z_{-2}}, \frac{A_{0}}{z_{-2}}\right\}=\frac{A_{0}}{z_{-2}}, \\
& z_{8}=\max \left\{\frac{A_{7}}{z_{7}}, z_{4}\right\}=\max \left\{\frac{A_{1} z_{-2}}{A_{0}}, z_{0}\right\}=z_{0}, \\
& z_{9}=\max \left\{\frac{A_{8}}{z_{8}}, z_{5}\right\}=\max \left\{\frac{A_{0}}{z_{0}}, z_{-3}\right\}=z_{-3},
\end{aligned}
$$

which is subsequently periodic with period four.

$$
\left\{z_{0}, z_{-3}, z_{-2}, \frac{A_{0}}{z_{-2}}, z_{0}, z_{-3}, \ldots\right\} \text {. }
$$

$\left(A_{2212}\right):$ if $A_{0} z_{0}<A_{1} z_{-2}$, then $z_{4}=A_{1} z_{-2} / A_{0}$ and

$$
\begin{aligned}
& z_{5}=\max \left\{\frac{A_{4}}{z_{4}}, z_{1}\right\}=\max \left\{\frac{A_{0}^{2}}{A_{1} z_{-2}}, z_{-3}\right\}=z_{-3}, \\
& z_{6}=\max \left\{\frac{A_{5}}{z_{5}}, z_{2}\right\}=\max \left\{\frac{A_{1}}{z_{-3}}, z_{-2}\right\}=z_{-2}, \\
& z_{7}=\max \left\{\frac{A_{6}}{z_{6}}, z_{3}\right\}=\max \left\{\frac{A_{0}}{z_{-2}}, \frac{A_{0}}{z_{-2}}\right\}=\frac{A_{0}}{z_{-2}}, \\
& z_{8}=\max \left\{\frac{A_{7}}{z_{7}}, z_{4}\right\}=\max \left\{\frac{A_{1} z_{-2}}{A_{0}}, \frac{A_{1} z_{-2}}{A_{0}}\right\}=\frac{A_{1} z_{-2}}{A_{0}}, \\
& z_{9}=\max \left\{\frac{A_{8}}{z_{8}}, z_{5}\right\}=\max \left\{\frac{A_{0}}{z_{0}}, z_{-3}\right\}=z_{-3},
\end{aligned}
$$

which is subsequently periodic with period four.

$$
\left\{\frac{A_{1} z_{-2}}{A_{0}}, z_{-3}, z_{-2}, \frac{A_{0}}{z_{-2}}, \frac{A_{1} z_{-2}}{A_{0}}, z_{-3}, \ldots\right\} .
$$

$\left(A_{222}\right)$ : if $A_{0}<z_{-2} z_{-1}$, then $z_{3}=z_{-1}$ and

$$
\begin{aligned}
& z_{4}=\max \left\{\frac{A_{3}}{z_{3}}, z_{0}\right\}=\max \left\{\frac{A_{1}}{z_{-1}}, z_{0}\right\}=z_{0}, \\
& z_{5}=\max \left\{\frac{A_{4}}{z_{4}}, z_{1}\right\}=\max \left\{\frac{A_{0}}{z_{0}}, z_{-3}\right\}=z_{-3} .
\end{aligned}
$$

Since, $z_{0} z_{-1}>A_{0}>A_{1}$, 


$$
z_{6}=\max \left\{\frac{A_{5}}{z_{5}}, z_{2}\right\}=\max \left\{\frac{A_{1}}{z_{-3}}, z_{-2}\right\} .
$$

$\left(A_{2221}\right):$ if $A_{1}<z_{-3} z_{-2}$, then $z_{6}=z_{-2}$ and

$$
\begin{aligned}
& z_{7}=\max \left\{\frac{A_{6}}{z_{6}}, z_{3}\right\}=\max \left\{\frac{A_{0}}{z_{-2}}, z_{-1}\right\}=z_{-1}, \\
& z_{8}=\max \left\{\frac{A_{7}}{z_{7}}, z_{4}\right\}=\max \left\{\frac{A_{1}}{z_{-1}}, z_{0}\right\}=z_{0}, \\
& z_{9}=\max \left\{\frac{A_{8}}{z_{8}}, z_{5}\right\}=\max \left\{\frac{A_{0}}{z_{0}}, z_{-3}\right\}=z_{-3}, \\
& z_{10}=\max \left\{\frac{A_{9}}{z_{9}}, z_{6}\right\}=\max \left\{\frac{A_{0}}{z_{-3}}, z_{-2}\right\}=z_{-2},
\end{aligned}
$$

which is subsequently periodic with period four.

$$
\left\{z_{-3}, z_{-2}, z_{-1}, z_{0}, z_{-3}, z_{-2}, \ldots\right\} \text {. }
$$

$\left(A_{2222}\right):$ if $A_{1}>z_{-3} z_{-2}$, then $z_{6}=A_{1} / z_{-3}$ and

$$
z_{7}=\max \left\{\frac{A_{6}}{z_{6}}, z_{3}\right\}=\max \left\{\frac{A_{0} z_{-3}}{A_{1}}, z_{-1}\right\}=\frac{A_{0} z_{-3}}{A_{1}} \text {. }
$$

Since, $z_{0} z_{-1}<A_{1}<A_{0}$,

$$
\begin{gathered}
z_{8}=\max \left\{\frac{A_{7}}{z_{7}}, z_{4}\right\}=\max \left\{\frac{A_{1}^{2}}{A_{0} z_{-3}}, z_{0}\right\}=\frac{A_{1}^{2}}{A_{0} z_{-3}} \\
z_{9}=\max \left\{\frac{A_{8}}{z_{8}}, z_{5}\right\}=\max \left\{\frac{A_{0}^{2} z_{-3}}{A_{1}^{2}}, z_{-3}\right\}=\frac{A_{0}^{2} z_{-3}}{A_{1}^{2}}, \\
z_{10}=\max \left\{\frac{A_{9}}{z_{9}}, z_{6}\right\}=\max \left\{\frac{A_{1}^{3}}{A_{0}^{2} z_{-3}}, \frac{A_{1}}{z_{-3}}\right\}=\frac{A_{1}}{z_{-3}},
\end{gathered}
$$

which is subsequently periodic with period four.

$$
\left\{\frac{A_{1}}{z_{-3}}, \frac{A_{0} z_{-3}}{A_{1}}, \frac{A_{1}^{2}}{A_{0} z_{-3}}, \frac{A_{0}^{2} z_{-3}}{A_{1}^{2}}, \frac{A_{1}}{z_{-3}}, \ldots\right\} .
$$

Theorem 5. Consider the difference equation (9), where $A_{0}<A_{1}$, Then, at some point, every solution of equation (9) is periodic with period four.

Proof. From (9), we have

$$
z_{1}=\max \left\{\frac{A_{0}}{z_{0}}, z_{-3}\right\} \text {. }
$$

We consider the following two cases:

Case $\left(A_{1}\right):$ if $z_{-3} z_{0}<A_{0}$, then $z_{1}=A_{0} / z_{0}$ and

$$
z_{2}=\max \left\{\frac{A_{1}}{z_{1}}, z_{-2}\right\}=\max \left\{\frac{A_{1} z_{0}}{A_{0}}, z_{-2}\right\} \text {. }
$$

$\left(A_{11}\right):$ if $A_{0} z_{-2}<A_{1} z_{0}$, then $z_{2}=A_{1} z_{0} / A_{0}$ and

$$
z_{3}=\max \left\{\frac{A_{2}}{z_{2}}, z_{-1}\right\}=\max \left\{\frac{A_{0}^{2}}{A_{1} z_{0}}, z_{-1}\right\} .
$$

$\left(A_{111}\right):$ if $A_{1} z_{-1} z_{0}<A_{0}^{2}$, then $z_{3}=A_{0}^{2} / A_{1} z_{0}$ and

$$
z_{4}=\max \left\{\frac{A_{3}}{z_{3}}, z_{0}\right\}=\max \left\{\frac{A_{1}^{2} z_{0}}{A_{0}^{2}}, z_{0}\right\} \text {. }
$$

$\left(A_{1111}\right)$ : if $A_{1}^{2} z_{0}>A_{0}^{2} z_{0}$, then $z_{4}=A_{1}^{2} z_{0} / A_{0}^{2}$ and

$$
\begin{aligned}
& z_{5}=\max \left\{\frac{A_{4}}{z_{4}}, z_{1}\right\}=\max \left\{\frac{A_{0}^{3}}{A_{1}^{2} z_{0}}, \frac{A_{0}}{z_{0}}\right\}=\frac{A_{0}}{z_{0}}, \\
& z_{6}=\max \left\{\frac{A_{5}}{z_{5}}, z_{2}\right\}=\max \left\{\frac{A_{1} z_{0}}{A_{0}}, \frac{A_{1} z_{0}}{A_{0}}\right\}=\frac{A_{1} z_{0}}{A_{0}}, \\
& z_{7}=\max \left\{\frac{A_{6}}{z_{6}}, z_{3}\right\}=\max \left\{\frac{A_{0}^{2}}{A_{1} z_{0}}, \frac{A_{0}^{2}}{A_{1} z_{0}}\right\}=\frac{A_{0}^{2}}{A_{1} z_{0}}, \\
& z_{8}=\max \left\{\frac{A_{7}}{z_{7}}, z_{4}\right\}=\max \left\{\frac{A_{1}^{2} z_{0}}{A_{0}^{2}}, \frac{A_{1}^{2} z_{0}}{A_{0}^{2}}\right\}=\frac{A_{1}^{2} z_{0}}{A_{0}^{2}}, \\
& z_{9}=\max \left\{\frac{A_{8}}{z_{8}}, z_{5}\right\}=\max \left\{\frac{A_{0}^{3}}{A_{1}^{2} z_{0}}, \frac{A_{0}}{z_{0}}\right\}=\frac{A_{0}}{z_{0}},
\end{aligned}
$$

which is subsequently periodic with period four.

$$
\left\{\frac{A_{1}^{2} z_{0}}{A_{0}^{2}}, \frac{A_{0}}{z_{0}}, \frac{A_{1} z_{0}}{A_{0}}, \frac{A_{0}^{2}}{A_{1} z_{0}}, \frac{A_{1}^{2} z_{0}}{A_{0}^{2}}, \frac{A_{0}}{z_{0}}, \ldots\right\} .
$$

$\left(A_{1112}\right):$ if $A_{1}^{2} z_{0}<A_{0}^{2} z_{0}$, then $z_{4}=z_{0}$ and

$$
\begin{aligned}
& z_{5}=\max \left\{\frac{A_{4}}{z_{4}}, z_{1}\right\}=\max \left\{\frac{A_{0}}{z_{0}}, \frac{A_{0}}{z_{0}}\right\}=\frac{A_{0}}{z_{0}}, \\
& z_{6}=\max \left\{\frac{A_{5}}{z_{5}}, z_{2}\right\}=\max \left\{\frac{A_{1} z_{0}}{A_{0}}, \frac{A_{1} z_{0}}{A_{0}}\right\}=\frac{A_{1} z_{0}}{A_{0}}, \\
& z_{7}=\max \left\{\frac{A_{6}}{z_{6}}, z_{3}\right\}=\max \left\{\frac{A_{0}^{2}}{A_{1} z_{0}}, \frac{A_{0}^{2}}{A_{1} z_{0}}\right\}=\frac{A_{0}^{2}}{A_{1} z_{0}}, \\
& z_{8}=\max \left\{\frac{A_{7}}{z_{7}}, z_{4}\right\}=\max \left\{\frac{A_{1}^{2} z_{0}}{A_{0}^{2}}, z_{0}\right\}=z_{0}, \\
& z_{9}=\max \left\{\frac{A_{8}}{z_{8}}, z_{5}\right\}=\max \left\{\frac{A_{0}^{3}}{A_{1}^{2} z_{0}}, \frac{A_{0}}{z_{0}}\right\}=\frac{A_{0}}{z_{0}},
\end{aligned}
$$

which is subsequently periodic with period four

$$
\left\{z_{0}, \frac{A_{0}}{z_{0}}, \frac{A_{1} z_{0}}{A_{0}}, \frac{A_{0}^{2}}{A_{1} z_{0}}, z_{0}, \frac{A_{0}}{z_{0}}, \ldots\right\} .
$$

$\left(A_{112}\right):$ if $A_{1} z_{-1} z_{0}>A_{0}^{2}$, then $z_{3}=z_{-1}$ and

$$
z_{4}=\max \left\{\frac{A_{3}}{z_{3}}, z_{0}\right\}=\max \left\{\frac{A_{1}}{z_{-1}}, z_{0}\right\} \text {. }
$$

$\left(A_{1121}\right):$ if $A_{1}>z_{-1} z_{0}$, then $z_{4}=A_{1} / z_{-1}$ and 


$$
\begin{aligned}
& z_{5}=\max \left\{\frac{A_{4}}{z_{4}}, z_{1}\right\}=\max \left\{\frac{A_{0} z_{-1}}{A_{1}}, \frac{A_{0}}{z_{0}}\right\}=\frac{A_{0}}{z_{0}}, \\
& z_{6}=\max \left\{\frac{A_{5}}{z_{5}}, z_{2}\right\}=\max \left\{\frac{A_{1} z_{0}}{A_{0}}, \frac{A_{1} z_{0}}{A_{0}}\right\}=\frac{A_{1} z_{0}}{A_{0}}, \\
& z_{7}=\max \left\{\frac{A_{6}}{z_{6}}, z_{3}\right\}=\max \left\{\frac{A_{0}^{2}}{A_{1} z_{0}}, z_{-1}\right\}=z_{-1} \text {, } \\
& z_{8}=\max \left\{\frac{A_{7}}{z_{7}}, z_{4}\right\}=\max \left\{\frac{A_{1}}{z_{-1}}, \frac{A_{1}}{z_{-1}}\right\}=\frac{A_{1}}{z_{-1}} \\
& z_{9}=\max \left\{\frac{A_{8}}{z_{8}}, z_{5}\right\}=\max \left\{\frac{A_{0} z_{-1}}{A_{1}}, \frac{A_{0}}{z_{0}}\right\}=\frac{A_{0}}{z_{0}}, \\
& z_{7}=\max \left\{\frac{A_{6}}{z_{6}}, z_{3}\right\}=\max \left\{\frac{A_{0}^{2}}{A_{1} z_{0}}, \frac{A_{0}}{z_{-2}}\right\}=\frac{A_{0}}{z_{-2}}, \\
& z_{8}=\max \left\{\frac{A_{7}}{z_{7}}, z_{4}\right\}=\max \left\{\frac{A_{1} z_{-2}}{A_{0}}, \frac{A_{1} z_{-2}}{A_{0}}\right\}=\frac{A_{1} z_{-2}}{A_{0}}, \\
& z_{9}=\max \left\{\frac{A_{8}}{z_{8}}, z_{5}\right\}=\max \left\{\frac{A_{0}^{2}}{A_{1} z_{-2}}, \frac{A_{0}}{z_{0}}\right\}=\frac{A_{0}}{z_{0}},
\end{aligned}
$$

which is subsequently periodic with period four.

$$
\left\{\frac{A_{1} z_{-2}}{A_{0}}, \frac{A_{0}}{z_{0}}, \frac{A_{1} z_{0}}{A_{0}}, \frac{A_{0}}{z_{-2}}, \frac{A_{1} z_{-2}}{A_{0}}, \frac{A_{0}}{z_{0}}, \ldots\right\} .
$$

$\left(A_{1212}\right)$ : if $A_{1} z_{-2}<A_{0} z_{0}$, then $z_{4}=z_{0}$ and

$\left(A_{1122}\right):$ if $A_{1}<z_{-1} z_{0}$, then $z_{4}=z_{0}$ and

$$
\begin{aligned}
& z_{5}=\max \left\{\frac{A_{4}}{z_{4}}, z_{1}\right\}=\max \left\{\frac{A_{0} z_{-1}}{A_{1}}, \frac{A_{0}}{z_{0}}\right\}=\frac{A_{0}}{z_{0}}, \\
& z_{6}=\max \left\{\frac{A_{5}}{z_{5}}, z_{2}\right\}=\max \left\{\frac{A_{1} z_{0}}{A_{0}}, \frac{A_{1} z_{0}}{A_{0}}\right\}=\frac{A_{1} z_{0}}{A_{0}}, \\
& z_{7}=\max \left\{\frac{A_{6}}{z_{6}}, z_{3}\right\}=\max \left\{\frac{A_{0}^{2}}{A_{1} z_{0}}, z_{-1}\right\}=z_{-1}, \\
& z_{8}=\max \left\{\frac{A_{7}}{z_{7}}, z_{4}\right\}=\max \left\{\frac{A_{1}}{z_{-1}}, \frac{A_{1}}{z_{-1}}\right\}=z_{0}, \\
& z_{9}=\max \left\{\frac{A_{8}}{z_{8}}, z_{5}\right\}=\max \left\{\frac{A_{0} z_{-1}}{A_{1}}, \frac{A_{0}}{z_{0}}\right\}=\frac{A_{0}}{z_{0}},
\end{aligned}
$$

$$
\begin{aligned}
& z_{5}=\max \left\{\frac{A_{4}}{z_{4}}, z_{1}\right\}=\max \left\{\frac{A_{0}}{z_{0}}, \frac{A_{0}}{z_{0}}\right\}=\frac{A_{0}}{z_{0}}, \\
& z_{6}=\max \left\{\frac{A_{5}}{z_{5}}, z_{2}\right\}=\max \left\{\frac{A_{1} z_{0}}{A_{0}}, z_{-2}\right\}=z_{-2}, \\
& z_{7}=\max \left\{\frac{A_{6}}{z_{6}}, z_{3}\right\}=\max \left\{\frac{A_{0}}{z_{-2}}, \frac{A_{0}}{z_{-2}}\right\}=\frac{A_{0}}{z_{-2}}, \\
& z_{8}=\max \left\{\frac{A_{7}}{z_{7}}, z_{4}\right\}=\max \left\{\frac{A_{1} z_{-2}}{A_{0}}, z_{0}\right\}=z_{0}, \\
& z_{9}=\max \left\{\frac{A_{8}}{z_{8}}, z_{5}\right\}=\max \left\{\frac{A_{0}}{z_{0}}, \frac{A_{0}}{z_{0}}\right\}=\frac{A_{0}}{z_{0}},
\end{aligned}
$$

which is subsequently periodic with period four.

$$
\left\{z_{0}, \frac{A_{0}}{z_{0}}, z_{-2}, \frac{A_{0}}{z_{-2}}, z_{0}, \frac{A_{0}}{z_{0}}, \ldots\right\} .
$$

$\left(A_{122}\right)$ : if $A_{0}<z_{-2} z_{-1}$, then $z_{3}=z_{-1}$ and

$$
z_{4}=\max \left\{\frac{A_{3}}{z_{3}}, z_{0}\right\}=\max \left\{\frac{A_{1}}{z_{-1}}, z_{0}\right\}=z_{0} \text {. }
$$

$$
z_{3}=\max \left\{\frac{A_{2}}{z_{2}}, z_{-1}\right\}=\max \left\{\frac{A_{0}^{2}}{A_{1} z_{0}}, z_{-1}\right\} \text {. }
$$

$\left(A_{121}\right)$ : if $A_{0}>z_{-2} z_{-1}$, then $z_{3}=A_{0} / z_{-2}$ and

$$
z_{4}=\max \left\{\frac{A_{3}}{z_{3}}, z_{0}\right\}=\max \left\{\frac{A_{1} z_{-2}}{A_{0}}, z_{0}\right\} \text {. }
$$

$\left(A_{1211}\right):$ if $A_{1} z_{-2}>A_{0} z_{0}$, then $z_{4}=A_{1} z_{-2} / A_{0}$ and

$$
\begin{aligned}
& z_{5}=\max \left\{\frac{A_{4}}{z_{4}}, z_{1}\right\}=\max \left\{\frac{A_{0}}{z_{0}}, \frac{A_{0}}{z_{0}}\right\}=\frac{A_{0}}{z_{0}}, \\
& z_{6}=\max \left\{\frac{A_{5}}{z_{5}}, z_{2}\right\}=\max \left\{\frac{A_{1} z_{0}}{A_{0}}, z_{-2}\right\}=z_{-2} .
\end{aligned}
$$


$\left(A_{1221}\right):$ if $A_{1} z_{0}>A_{0} z_{-2}$, then $z_{6}=A_{1} z_{0} / A_{0}$ and

$z_{7}=\max \left\{\frac{A_{6}}{z_{6}}, z_{3}\right\}=\max \left\{\frac{A_{0}^{2}}{A_{1} z_{0}}, z_{-1}\right\}=\frac{A_{0}^{2}}{A_{1} z_{0}}$.

Since, $A_{1} z_{-1} z_{0}<A_{0}^{2}$,

$$
\begin{aligned}
& z_{8}=\max \left\{\frac{A_{7}}{z_{7}}, z_{4}\right\}=\max \left\{\frac{A_{1}^{2} z_{0}}{A_{0}^{2}}, z_{0}\right\}=\frac{A_{1}^{2} z_{0}}{A_{0}^{2}}, \\
& z_{9}=\max \left\{\frac{A_{8}}{z_{8}}, z_{5}\right\}=\max \left\{\frac{A_{0}^{3}}{A_{1}^{2} z_{0}}, \frac{A_{0}}{z_{0}}\right\}=\frac{A_{0}}{z_{0}},
\end{aligned}
$$

which is subsequently periodic with period four.

$$
\left\{\frac{A_{0}}{z_{0}}, \frac{A_{1} z_{0}}{A_{0}}, \frac{A_{0}^{2}}{A_{1} z_{0}}, \frac{A_{1}^{2} z_{0}}{A_{0}^{2}}, \frac{A_{0}}{z_{0}}, \ldots\right\} .
$$

$\left(A_{1222}\right):$ if $A_{1} z_{0}<A_{0} z_{-2}$, then $z_{6}=z_{-2}$ and

$$
z_{7}=\max \left\{\frac{A_{6}}{z_{6}}, z_{3}\right\}=\max \left\{\frac{A_{0}}{z_{-2}}, z_{-1}\right\}=\frac{A_{0}}{z_{-2}} .
$$

Since, $A_{1} z_{-1} z_{0}<A_{0}^{2}$,

$$
\begin{aligned}
& z_{8}=\max \left\{\frac{A_{7}}{z_{7}}, z_{4}\right\}=\max \left\{\frac{A_{1} z_{-2}}{A_{0}}, z_{0}\right\}=z_{0}, \\
& z_{9}=\max \left\{\frac{A_{8}}{z_{8}}, z_{5}\right\}=\max \left\{\frac{A_{0}}{z_{0}}, \frac{A_{0}}{z_{0}}\right\}=\frac{A_{0}}{z_{0}},
\end{aligned}
$$

which is subsequently periodic with period four.

$$
\left\{z_{-2}, \frac{A_{0}}{z_{-2}}, z_{0}, \frac{A_{0}}{z_{0}}, z_{-2}, \ldots\right\} \text {. }
$$

Case $\left(A_{2}\right):$ if $z_{-3} z_{0}>A_{0}$, then $z_{1}=z_{-3}$ and

$$
z_{2}=\max \left\{\frac{A_{1}}{z_{1}}, z_{-2}\right\}=\max \left\{\frac{A_{1}}{z_{-3}}, z_{-2}\right\} .
$$

$\left(A_{21}\right):$ if $A_{1}>z_{-3} z_{-2}$, then $z_{2}=A_{1} / z_{-3}$ and

$$
z_{3}=\max \left\{\frac{A_{2}}{z_{2}}, z_{-1}\right\}=\max \left\{\frac{A_{0} z_{-3}}{A_{1}}, z_{-1}\right\} .
$$

$\left(A_{211}\right):$ if $A_{1} z_{-1}>A_{0} z_{-3}$, then $z_{3}=z_{-1}$ and

$$
\begin{aligned}
& z_{4}=\max \left\{\frac{A_{3}}{z_{3}}, z_{0}\right\}=\max \left\{\frac{A_{1}}{z_{-1}}, z_{0}\right\}=\frac{A_{1}}{z_{-1}}, \\
& z_{5}=\max \left\{\frac{A_{4}}{z_{4}}, z_{1}\right\}=\max \left\{\frac{A_{0} z_{-1}}{A_{1}}, z_{-3}\right\}=z_{-3}, \\
& z_{6}=\max \left\{\frac{A_{5}}{z_{5}}, z_{2}\right\}=\max \left\{\frac{A_{1}}{z_{-3}}, \frac{A_{1}}{z_{-3}}\right\}=\frac{A_{1}}{z_{-3}}, \\
& z_{7}=\max \left\{\frac{A_{6}}{z_{6}}, z_{3}\right\}=\max \left\{\frac{A_{0} z_{-3}}{A_{1}}, z_{-1}\right\}=z_{-1}, \\
& z_{8}=\max \left\{\frac{A_{7}}{z_{7}}, z_{4}\right\}=\max \left\{\frac{A_{1}}{z_{-1}}, \frac{A_{1}}{z_{-1}}\right\}=\frac{A_{1}}{z_{-1}}, \\
& z_{9}=\max \left\{\frac{A_{8}}{z_{8}}, z_{5}\right\}=\max \left\{\frac{A_{0} z_{-1}}{A_{1}}, z_{-3}\right\}=z_{-3},
\end{aligned}
$$

which is subsequently periodic with period four.

$$
\left\{z_{-1}, \frac{A_{1}}{z_{-1}}, z_{-3}, \frac{A_{1}}{z_{-3}}, z_{-1}, \frac{A_{1}}{z_{-1}}, z_{-3}, \ldots\right\} .
$$

$\left(A_{212}\right):$ if $A_{1} z_{-1}<A_{0} z_{-3}$, then $z_{3}=A_{0} z_{-3} / A_{1}$ and

$$
\begin{aligned}
& z_{4}=\max \left\{\frac{A_{3}}{z_{3}}, z_{0}\right\}=\max \left\{\frac{A_{1}^{2}}{A_{0} z_{-3}}, z_{0}\right\}=\frac{A_{1}^{2}}{A_{0} z_{-3}}, \\
& z_{5}=\max \left\{\frac{A_{4}}{z_{4}}, z_{1}\right\}=\max \left\{\frac{A_{0} z_{-3}}{A_{1}}, z_{-3}\right\}=z_{-3}, \\
& z_{6}=\max \left\{\frac{A_{5}}{z_{5}}, z_{2}\right\}=\max \left\{\frac{A_{1}}{z_{-3}}, \frac{A_{1}}{z_{-3}}\right\}=\frac{A_{1}}{z_{-3}}, \\
& z_{7}=\max \left\{\frac{A_{6}}{z_{6}}, z_{3}\right\}=\max \left\{\frac{A_{0} z_{-3}}{A_{1}}, \frac{A_{0} z_{-3}}{A_{1}}\right\}=\frac{A_{0} z_{-3}}{A_{1}}, \\
& z_{9}=\max \left\{\frac{A_{7}}{z_{7}}, z_{4}\right\}=\max \left\{\frac{A_{1}^{2}}{A_{0} z_{-3}}, \frac{A_{1}^{2}}{A_{0} z_{-3}}\right\}=\frac{A_{1}^{2}}{A_{0} z_{-3}}, \\
& \left.z_{5}\right\}=\max \left\{\frac{A_{0}^{2} z_{-3}}{A_{1}^{2}}, z_{-3}\right\}=z_{-3},
\end{aligned}
$$

which is subsequently periodic with period four.

$$
\left\{\frac{A_{0} z_{-3}}{A_{1}}, \frac{A_{1}^{2}}{A_{0} z_{-3}}, z_{-3}, \frac{A_{1}}{z_{-3}}, \frac{A_{0} z_{-3}}{A_{1}}, \frac{A_{1}^{2}}{A_{0} z_{-3}}, z_{-3}, \ldots\right\} .
$$

$\left(A_{22}\right):$ if $A_{1}<z_{-3} z_{-2}$, then $z_{2}=z_{-2}$ and 


$$
z_{3}=\max \left\{\frac{A_{2}}{z_{2}}, z_{-1}\right\}=\max \left\{\frac{A_{0}}{z_{-2}}, z_{-1}\right\} .
$$

$\left(A_{221}\right)$ : if $A_{0}>z_{-2} z_{-1}$, then $z_{3}=A_{0} / z_{-2}$ and

$$
z_{4}=\max \left\{\frac{A_{3}}{z_{3}}, z_{0}\right\}=\max \left\{\frac{A_{1} z_{-2}}{A_{0}}, z_{0}\right\} \text {. }
$$

$\left(A_{2211}\right):$ if $A_{1} z_{-2}>A_{0} z_{0}$, then $z_{4}=A_{1} z_{-2} / A_{0}$ and $z_{5}=\max \left\{\frac{A_{4}}{z_{4}}, z_{1}\right\}=\max \left\{\frac{A_{0}^{2}}{A_{1} z_{-2}}, z_{-3}\right\}=z_{-3}$,

$$
\begin{aligned}
& z_{6}=\max \left\{\frac{A_{5}}{z_{5}}, z_{2}\right\}=\max \left\{\frac{A_{1}}{z_{-3}}, z_{-2}\right\}=z_{-2}, \\
& z_{7}=\max \left\{\frac{A_{6}}{z_{6}}, z_{3}\right\}=\max \left\{\frac{A_{0}}{z_{-2}}, \frac{A_{0}}{z_{-2}}\right\}=\frac{A_{0}}{z_{-2}}
\end{aligned}
$$

$$
z_{8}=\max \left\{\frac{A_{7}}{z_{7}}, z_{4}\right\}=\max \left\{\frac{A_{1} z_{-2}}{A_{0}}, \frac{A_{1} z_{-2}}{A_{0}}\right\}=\frac{A_{1} z_{-2}}{A_{0}}
$$$$
z_{9}=\max \left\{\frac{A_{8}}{z_{8}}, z_{5}\right\}=\max \left\{\frac{A_{0}^{2}}{A_{1} z_{-2}}, z_{-3}\right\}=z_{-3},
$$

which is subsequently periodic with period four.

$$
\left\{\frac{A_{1} z_{-2}}{A_{0}}, z_{-3}, z_{-2}, \frac{A_{0}}{z_{-2}}, \frac{A_{1} z_{-2}}{A_{0}}, z_{-3}, \ldots\right\} \text {. }
$$

$\left(A_{2212}\right)$ : if $A_{1} z_{-2}<A_{0} z_{0}$, then $z_{4}=z_{0}$ and

$$
\begin{aligned}
& z_{5}=\max \left\{\frac{A_{4}}{z_{4}}, z_{1}\right\}=\max \left\{\frac{A_{0}}{z_{0}}, z_{-3}\right\}=\frac{A_{0}}{z_{0}} \\
& z_{6}=\max \left\{\frac{A_{5}}{z_{5}}, z_{2}\right\}=\max \left\{\frac{A_{1} z_{0}}{A_{0}}, z_{-2}\right\}=z_{-2}, \\
& z_{7}=\max \left\{\frac{A_{6}}{z_{6}}, z_{3}\right\}=\max \left\{\frac{A_{0}}{z_{-2}}, \frac{A_{0}}{z_{-2}}\right\}=\frac{A_{0}}{z_{-2}} \\
& z_{8}=\max \left\{\frac{A_{7}}{z_{7}}, z_{4}\right\}=\max \left\{\frac{A_{1} z_{-2}}{A_{0}}, z_{0}\right\}=z_{0}, \\
& z_{9}=\max \left\{\frac{A_{8}}{z_{8}}, z_{5}\right\}=\max \left\{\frac{A_{0}}{z_{0}}, \frac{A_{0}}{z_{0}}\right\}=\frac{A_{0}}{z_{0}}
\end{aligned}
$$

which is subsequently periodic with period four.

$$
\left\{z_{0}, \frac{A_{0}}{z_{0}}, z_{-2}, \frac{A_{0}}{z_{-2}}, z_{0}, \frac{A_{0}}{z_{0}}, \ldots\right\} .
$$

$\left(A_{222}\right):$ if $A_{0}<z_{-2} z_{-1}$, then $z_{3}=z_{-1}$ and

$$
\begin{aligned}
& z_{4}=\max \left\{\frac{A_{3}}{z_{3}}, z_{0}\right\}=\max \left\{\frac{A_{1}}{z_{-1}}, z_{0}\right\}=\frac{A_{1}}{z_{-1}}, \\
& z_{5}=\max \left\{\frac{A_{4}}{z_{4}}, z_{1}\right\}=\max \left\{\frac{A_{0} z_{-1}}{A_{1}}, z_{-3}\right\}=z_{-3}, \\
& z_{6}=\max \left\{\frac{A_{5}}{z_{5}}, z_{2}\right\}=\max \left\{\frac{A_{1}}{z_{-3}}, z_{-2}\right\} . \\
& \left(A_{2221}\right): \operatorname{if} A_{1}>z_{-3} z_{-1}, \operatorname{then} z_{7}=A_{1} / z_{-3} \text { and } \\
& z_{7}=\max \left\{\frac{A_{6}}{z_{6}}, z_{3}\right\}=\max \left\{\frac{A_{0} z_{-3}}{A_{1}}, z_{-1}\right\}=z_{-1}, \\
& z_{8}=\max \left\{\frac{A_{7}}{z_{7}}, z_{4}\right\}=\max \left\{\frac{A_{1}}{z_{-1}}, \frac{A_{1}}{z_{-1}}\right\}=\frac{A_{1}}{z_{-1}}, \\
& z_{9}=\max \left\{\frac{A_{8}}{z_{8}}, z_{5}\right\}=\max \left\{\frac{A_{0} z_{-1}}{A_{1}}, z_{-3}\right\}=z_{-3},
\end{aligned}
$$

which is subsequently periodic with period four.

$$
\left\{\frac{A_{1}}{z_{-3}}, z_{-1}, \frac{A_{1}}{z_{-1}}, z_{-3}, \frac{A_{1}}{z_{-1}}, \ldots\right\} .
$$

$\left(A_{2222}\right):$ if $A_{1}<z_{-3} z_{-1}$, then $z_{7}=z_{-2}$ and

$$
\begin{aligned}
& z_{7}=\max \left\{\frac{A_{6}}{z_{6}}, z_{3}\right\}=\max \left\{\frac{A_{0}}{z_{-2}}, z_{-1}\right\}=\frac{A_{0}}{z_{-2}}, \\
& z_{8}=\max \left\{\frac{A_{7}}{z_{7}}, z_{4}\right\}=\max \left\{\frac{A_{1} z_{-2}}{A_{0}}, \frac{A_{1}}{z_{-1}}\right\}=\frac{A_{1}}{z_{-1}}, \\
& z_{9}=\max \left\{\frac{A_{8}}{z_{8}}, z_{5}\right\}=\max \left\{\frac{A_{0} z_{-1}}{A_{1}}, z_{-3}\right\}=z_{-3},
\end{aligned}
$$

which is subsequently periodic with period four.

$$
\left\{z_{-2}, \frac{A_{0}}{z_{-2}}, \frac{A_{1}}{z_{-1}}, z_{-3}, z_{-2}, \ldots\right\} .
$$

\section{Conclusion}

In this paper, we investigate the dynamic behaviors of a class of discrete model (1) which is max-type difference equation model with period four. We take the following two cases $A_{1}$ and $A_{2}$ and made all the subcases with the help of these two cases. Case $A_{1}$ has twelve subcases and case $A_{2}$ has ten subcases in each theorem. We have discussed every subcase and found the solution under some conditions. We conclude that every dynamic behavior of discrete max-type difference equation is eventually periodic with some period. In future research, we will look at a model of the same sort of maximal (minimal) difference equations with arbitrary real numbers as parameters so that more common phenomena can be studied and analyzed and conclusions can be drawn [35-37]. 


\section{Data Availability}

All data used in the manuscript are properly cited in the article.

\section{Conflicts of Interest}

The authors declare that they have no conflicts of interest.

\section{Acknowledgments}

This article was funded by the Deanship of Scientific Research (DSR), University of Tabuk. The authors, therefore, acknowledge with thanks DSR technical and financial support.

\section{References}

[1] S. Kanaun, "Discrete model of hydraulic fracture crack propagation in homogeneous isotropic elastic media," International Journal of Engineering Science, vol. 110, pp. 1-14, 2017.

[2] A. U. Rehman, C. Dong, V. A. Thomas, L. L. Yang, and H. Lajos, "Throughput and delay analysis of cognitive goback-N hybrid automatic repeat reQuest using discrete-time markov modelling," IEEE Access, vol. 4, no. 99, pp. 9659-9680, 2016.

[3] Z. Wei, W. Hongbin, W. Hongrui, S. Wen, and Z. M. Zhang, "Fuzzy dynamic output feedback control for T-S fuzzy discretetime systems with multiple time-varying delays and unmatched disturbances," IEEE Access, vol. 6, pp. 31037-31049, 2018.

[4] Y. Luo, S. Gao, D. Xie, and Y. Dai, "A discrete plant disease model with roguing and repanting," Advances in Difference Equations, vol. 2015, no. 1, pp. 1-18, 2015.

[5] H. El-Metwally, "Global behavior of an economic model," Chaos, Solitons \& Fractals, vol. 33, no. 3, pp. 994-1005, 2007.

[6] F. Mohammadi, "A computational approach for solution of boundary layer equations for the free convection along a vertical plate," Journal of Computational Methods in Science and Engineering, vol. 15, no. 3, pp. 317-326, 2015.

[7] M. Mez and A. N. Luiggi, "Adapting the condensation and evaporation model to the study of kinetics of phase transformations in binary metal systems," Journal of Computational Methods in Science and Engineering, vol. 14, no. 1-3, pp. 179-194, 2014.

[8] H. El-Metwally and M. M. El-Afifi, "On the behavior of some extension forms of some population Models," Chaos, Solitons \& Fractals, vol. 36, no. 1, pp. 104-114, 2008.

[9] L. Zhou, H. U. Honghua, C. Liang et al., "SoResearch on difference equation model in traffic flow calculation," Journal of Chang chun University of Science and Technology, vol. 2, no. 2014, pp. 117-123, 2014.

[10] C. M. Huang and W. P. Wang, "Applications of difference equation in population forecasting model," Advanced Materials Research, vol. 1079-1080, no. 1-2, pp. 664-667, 2015.

[11] N. Touafek and E. M. Elsayed, "On the solutions of systems of rational difference equations," Mathematical and Computer Modelling, vol. 55, no. 7-8, pp. 1987-1997, 2012.

[12] C. Flaut, "Some application of difference equations in cryptography and coding theory," Journal of Difference Equations and Applications, vol. 25, no. 2, pp. 1-18, 2018.
[13] L. A. Zadeh, "Toward a generalized theory of uncertainty (GTU)-An outline," Information Sciences, vol. 172, no. 1, pp. 1-40, 2005.

[14] A. Mozaffari and N. L. Azad, "An ensemble neuro-fuzzy radial basis network with self-adaptive swarm based supervisor and negative correlation for modeling automotive engine coldstart hydrocarbon emissions: a soft solution to a crucial automotive problem," Applied Soft Computing, vol. 32, pp. 449-467, 2015.

[15] N. Li, D. B. Sun, T. Zou, and Y. Q. Qin, "An analysis for a particle's trajectory of PSO based on difference equation," Chinese Journal of Computers, vol. 29, no. 11, pp. 2052-2060, 2006.

[16] Z. M. Zhu, R. Q. Liu, F. F. Liu, and Y. Cao, "Analysis of image processing model based on pixels of solitary waves," Journal of Beijing University of Aeronautics and Astronautics, vol. 41, no. 12, pp. 2335-2339, 2015.

[17] C.-m. Chen, C.-m. Hong, and H.-c. Chuang, "Efficient autofocus algorithm utilizing discrete difference equation prediction model for digital still cameras," IEEE Transactions on Consumer Electronics, vol. 52, no. 4, pp. 1135-1143, 2006.

[18] X. Fan, L. Wang L, and Z. Teng, "Global dynamics for a class of discrete SEIRS epidemic models with general nonlinear incidence," Advances in Difference Equations, vol. 2016, no. 1, pp. 1-20, 2016.

[19] J. L. Williams, "On a class of nonlinear max-type difference equations," Cogent Mathematics, vol. 3, no. 1, pp. 1-11, 2016.

[20] S. Stevi'c, "On the recursive sequence $x_{n+1}=A+x_{n}^{p} / x_{n-1}^{p}$," Discrete Dynamics in Nature and Society, vol. 2007, no. 2, pp. 1-9, 2014.

[21] F. Sun, "On the asymptotic behavior of a difference equation with maximum," Discrete Dynamics in Nature and Society, vol. 2008, no. 1-2, 6 pages, Article ID 2432291, 2008.

[22] S. Guangwang, S. Taixiang, and Q. Bin, "Eventually periodic solutions of a max-type system of difference equations of higher order," Discrete Dynamics in Nature and Society, vol. 2018, Article ID 8467682, 6 pages, 2018.

[23] X. Yang, X. Liao, and C. Li, "On a difference equation with maximum," Applied Mathematics and Computation, vol. 181, no. 1, pp. 1-5, 2006.

[24] A. Gelisken and C. Qinar, "On the global attractivity of a maxtype difference equation," Discrete Dynamics in Nature and Society, vol. 2009, no. 10-12, 5 pages, Article ID 812674, 2009.

[25] T. Sun, J. Liu, Q. He, X.-H. Liu, and C. Tao, "Eventually periodic solutions of a max-type difference equation," The Scientific World Journal, vol. 2014, Article ID 219437, 4 pages, 2014.

[26] C. Cinar and I. Yalcinkaya, "On the positive solutions of the difference equation $\mathrm{x} \_\{\mathrm{n}+1\}=\max \left\{\mathrm{A} / \mathrm{x} \_\mathrm{n} 2, \mathrm{Bx} \_\{\mathrm{n}-1\} /\left(\mathrm{x} \_\mathrm{nx} \_\right.\right.$ $\{\mathrm{n}-2\} 2)\} \quad x_{n+1}=\max \left\{A / x_{n}^{2}, B x_{n-1} / x_{n} \bar{x}_{n-2}^{2}\right\}, " \quad$ International Journal of Contemporary Mathematical Sciences, vol. 1, no. 10, pp. 489-494, 2006.

[27] E. M. Elsayed and S. Stević, "On the max-type equation," Nonlinear Analysis: Theory, Methods \& Applications, vol. 71, no. 3-4, pp. 910-922, 2009.

[28] E. M. Elsayed and B. D. Iričanin, "On a max-type and a mintype difference equation," Applied Mathematics and Computation, vol. 215, no. 2, pp. 608-614, 2009.

[29] D. Simsek, C. Cinar, and I. Yalcinkaya, "On the solutions of the difference equation $x_{-}\{n+1\}=\max \left\{1 / x_{-}\{n-1\}, x_{-}\{n-1\}\right\}$," International Journal of Contemporary Mathematical Sciences, vol. 1, no. 10, pp. 481-487, 2006.

[30] D. Simsek, B. Demir, and C. Cinar, "On the Solutions of the System of Difference Equationsxn $+1=\max \{\mathrm{A} / \mathrm{xn}, \mathrm{yn} /$ 
$\mathrm{xn}\}, \mathrm{yn}+1=\max \{\mathrm{A} / \mathrm{yn}, \mathrm{xn} / \mathrm{yn}\}, "$ Discrete Dynamics in Nature and Society, vol. 2009, Article ID 325296, 11 pages, 2009.

[31] S. Stevic, "On the recursive sequence $x_{n+1}=\max \left\{C, x_{n}^{p} / x_{n-1}^{p}\right\}, "$ Applied Mathematics Letters, vol. 21, no. 8, pp. 791-796, 2008.

[32] I. Yalcinkaya, C. Cinar, and A. Gelisken, "On the recursive sequence," Discrete Dynamics in Nature and Society, vol. 2010, Article ID 583230, 13 pages, 2010.

[33] D. M. Chan, C. M. Kent, V. Kocić, and S. Stević, "A proposal for an application of a max-type difference equation to epilepsy," in Proceedings of the Differential and Difference Equations with Applications. ICDDEA 2017, S. Pinelas, T. Caraballo, P. Kloeden, and J. Graef, Eds., Cham, June 2018.

[34] M. C. Corballis, The Recursive Mind: The Origins of Human Language, Thought, and Civilization, Princeton University Press, Princeton, NJ, USA, 2011.

[35] M. R. S. Kulenovic and G. Ladas, Dynamics of Second Order Rational Difference Equations: With Open Problems and Conjectures, Chapman Hall/CRC, New York, NY, USA, 2002.

[36] E. A. Grove and G. Ladas, Periodicities in Nonlinear Difference Equations, vol. 4, Chaman and hall/CRC, New York, NY, USA, 2005.

[37] C. Wang and J. Li, "Periodic solution for a max-type fuzzy difference equation," Journal of Mathematics, vol. 2020, Article ID 3094391, 12 pages, 2020. 